AUTOMORPHISM INVARIANTS FOR SEMIGROUPS

by

W. Barit

No. 38

October, 1985 


\title{
AUTOMORPHISM INVARIANTS FOR SEMIGROUPS
}

\author{
William Barit
}

Invariant subgroups are associated with each element of a semigroup. These invariants are used to show certain semigroups of continuous functions have only inner automorphisms. In special cases bijections preserving these invariants are necessarily automorphisms and outer automorphisms can be constructed.

\section{BACKGROUND}

Fitzpatrick and symons [3] showed that if $x$ is an infinite set, and $S$ any semigroup of total transformations which contain $G$, the symmetric group on $x$, then all automorphisms of $S$ are inner (i.e. $S$ has the inner automorphism property, i.a.p.). Wood [11] produced general results in the topological case for $x=I$, the unit interval. He was able to show that certain semigroups of continuous transformations on $x$ containing the group of homeomorphisms have the i.a.p., and in fact characterize the automorphisms of semigroups containing both the group and constants. As with the Fitzpatrick and symons results, the fact that the group of homeomorphisms have the i.a.p. was used (Fine \& Schweigert [2]). Whittaker [10] generalized this to a class of spaces called regionally Euclidean (which includes manifolds). This paper deals with compact manifolds and extends the results of wood to characterize a wider class of semigroups as having the i.a.p.

Other related results include sullivan [9] who deals with certain semigroups of partial transformations on a set, Gluskin $[4,5]$ who dealt with certain specific semigroups on closed bounded subsets of $\mathrm{R}^{\mathrm{n}}$, and Magill [7] who dealt with endomorphisms on the full semigroup of continuous maps for a wide class of spaces. 


\section{INTRODUCTION}

Let $T$ be a transformation semigroup on $x, G$ be the group of invertible elements of $T$, and $S$ be a semigroup of $T$ which contains $G$. In this paper, results are given in two settings, first when $T$ is the full transformation semigroup on the set $x$, so $G$ is the group of bijections on $x$, and second when $T$ is the semigroup of continuous maps on a compact manifold; $\mathrm{x}$, so $G$ is the group of homeomorphisms. Other settings that are appropriate to the techniques presented here are continuous functions on more general spaces (e.g. Regionally Euclidean), and differentiable functions.

DEFINITION 1.1. Inn $(S)$ is the set of all automorphisms of $S$ produced by conjugation by elements of $G$.

DEFINITION 1.2. Gid $(S)$ is the set of all automorphisms of $S$ which when restricted to $G$ are the identity.

It is sufficient to consider only these when studying automorphisms on $S$, since the restriction to $G$ of any automorphisms of $S$ is inner. (First used by Fitzpatrick and Symons). Further such $\phi$ are inner if and only if $\phi=i d$. This follows from the fact that the Center of $G$ is $\{i d\}$. This is a consequence of the 2-transitivity of $G$. In fact we get

PROPOSITION 1.3. If the Center $(G)=\{i d\}, G$ has the iap, and $S$ is a semigroup $\supset G$, then Inn $(S) \triangleleft$ Aut $(S)$, Gid $(S) \triangleleft$ Aut $(S)$, and any element from Gid (S) commutes with any from Inn (S).

Proof. Take $\psi \in \operatorname{Inn}(S)$ so $\psi(f)=h_{f h}^{-1}$. Consider $\phi \psi \phi^{-1}(f)=$ $\phi\left(h \phi^{-1} f h^{-1}\right)=(\phi h)\left(\phi^{-1} \phi f\right)\left(\phi h^{-1}\right)=(\phi h) f(\phi h)^{-1}$ which is inner. Take $\psi^{\prime} \in \operatorname{Gid}(S)$. Now $\phi \psi^{\prime} \phi^{-1}(g)=\phi \psi^{\prime}\left(\phi^{-1} g\right)=\phi \phi^{-1} g=g$ so $\phi \psi^{\prime} \phi^{-1}$ is in Gid (S). Consider $\psi \psi^{\prime}(f)=h \psi^{\prime}(f) h^{-1}$ and $\psi^{\prime} \psi(f)=\psi^{\prime}\left(h f h^{-1}\right)=$ $h \psi^{\prime}(f) h^{-1}$.

Thus the following holds.

PROPOSITION 1.4. With conditions on $S$ and $G$ as above, Aut $(S) \cong$ Gid (S) $\times$ Inn (S).

For the rest of this paper. we consider only those automoxphisms of $S$ which are the identity on $G$, and use $\phi$ to denote them. 


\section{INVARIANT GROUPS}

DEFINITION 2.1 .

For $f$ in $S$

$$
\begin{aligned}
& L_{f}=\{g \text { in } G \mid g f=f\} \\
& R_{E}=\{g \text { in } G \mid f g=f\} \\
& I_{f}=\left\{\left(g_{1}, g_{2}\right) \text { in } G \times G \mid g_{1} f_{2}^{-1}=f\right\} .
\end{aligned}
$$

These are subgroups of $G$ or $G \times G$. Since $\phi$ preserves commuting diagrams, the same groups are identified to both $f$ and $\phi f$.

$\left(e \cdot g \cdot L_{f}=L_{\phi f}\right)$.

PROPOSITION 2.2. $L_{\mathrm{f}} \times \mathrm{R}_{\mathrm{f}}\left\langle\mathrm{I}_{\mathrm{f}} \cdot\right.$

Proof. Take $g_{1}^{\prime} \in L_{f}$ and $g_{2}^{\prime} \in R_{f_{1}^{\prime}} g_{1}^{\prime} f\left(g_{2}^{\prime}\right)^{-1}=f . \quad$ For $\left(g_{1}, g_{2}\right) \in I_{f}$ consider $g_{1}^{-1} g_{1}^{\prime} g_{1}$ and $g_{2}^{-1} g_{2}^{\prime} g_{2} . \quad g_{1}^{-1} g_{1}^{\prime} g_{1} f=g_{1}^{-1} g_{1}^{\prime} f_{2}$ $=g_{1}^{-1} \mathrm{fg}_{2}=\mathrm{f}$ so $\mathrm{g}_{1}^{-1} \mathrm{~g}_{1}^{\prime} \mathrm{g}_{1} \in \mathrm{L}_{\mathrm{f}}$ similarly $\mathrm{g}_{2}^{-1} \mathrm{~g}_{2}^{\prime} \mathrm{g}_{2} \in \mathrm{R}_{\mathrm{f}}$.

PROPOSITION 2.3. $\mathrm{R}_{\mathrm{fg}}=\mathrm{g}^{-1} \mathrm{R}_{\mathrm{f}} \mathrm{g}, \mathrm{L}_{\mathrm{gf}}=\mathrm{gL}_{\mathrm{f}} \mathrm{g}^{-1}$, and

$$
I_{g^{\prime} f g}=\left(g^{\prime}, g^{-1}\right) I_{f}\left(g^{\prime}, g^{-1}\right)^{-1}
$$

These invariants come about in the context of group action. For example $G \times G$ acts faithfully on $S$ by

$$
\left(g_{1}, g_{2}\right) \cdot f=g_{1} f g_{2}^{-1} \text {. }
$$

$I_{f}$ is the stabilizer of this action. A faithful action means the homomorphism $\Phi: G \times G$ to Bijections on $S$ is 1-1. In this case this follows from the fact that the Center of $G$ is trivial. Note also for all $\left(g_{1}, g_{2}\right), \Phi\left(g_{1}, g_{2}\right)$ commutes with $\phi$.

Intuitive viewpoint

The domain of a function $f$ is equivalent to its graph, so $f$ can be viewed as the projection of a "folded" version of $x$ (the graph of f) into an unfolded version of $x$ (the second axis). This viewpoint helps describe these invariants and illustrate some associated concepts.

EXAMPLE: Here $X=I^{2}$, the euclidean 2 cell. $S$ the semigroup of continuous functions. Consider the function for this picture

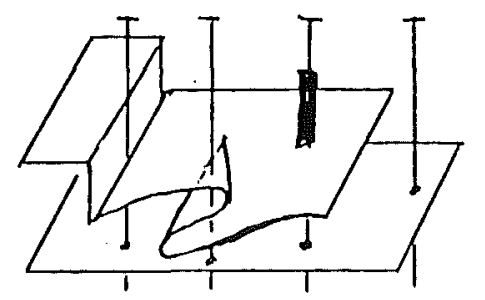


which illustrates

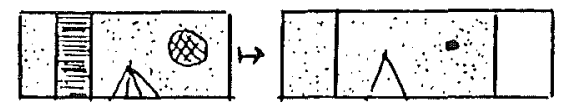

The vertical "pins" describe the function $f$. The top part of the pin is the inverse image of the value which is the bottom part of the pin. A pin attached to the top surface would be "bent" by a homeomorphism of the lower surface which moved the point where the bottom is attached, but pins piercing only the lower surface can be freely moved. Similarly some homeomorphisms of the top surface may not "bend" a pin if there is no horizontal motion but only vertical. movement within in the top of the pin. A pair of homeomorphism of the top and bottom surfaces may jointly move pins without bending them.

$I_{f}, R_{f}$, and $I_{f}$ respectively correspond to all homeomorphisms of the bottom, top, or both surfaces that move the pins without distortion. In the example illustrated:

$\mathrm{L}_{\mathrm{f}}-\mathrm{all}$ homeomorphisms id on ran $\mathrm{f}$

$\mathrm{R}_{\mathrm{f}}$ - all homeomorphisms id in sis:

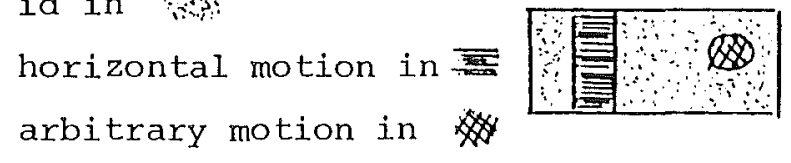

$I_{f}-a l l$ homeomorphisms of the lower surface with motion restricted to indicated set

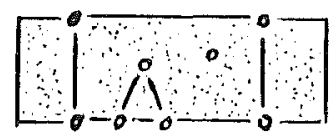

the homeomorphism of the top being determined up to type as in $R_{f}$.

\section{GEOMETRIC INVARIANTS}

It is possible to get invaxiant subsets in the following way.

DEFINITION 3.1 .

For $x$ in $x \quad \begin{aligned} L_{f}(x) & =\left\{g(x) \mid g \text { in } L_{f}\right\} \\ R_{f}(x) & =\left\{g(x) \mid g \text { in } R_{f}\right\}\end{aligned}$

For $x, y$ in $x \quad I_{f}(x, y)=\left\{\left(g_{2}(x), g_{1}(y)\right) \mid\left(g_{1}, g_{2}\right)\right.$ in $\left.I_{f}\right\}$ $K\left(L_{f}\right)=\left\{x\right.$ in $x \mid L_{f}(x)=\{x\}$

These invariants relate to group action as well. $G$ and $G \times G$ act faithfully on $x, x \times x$ respectively, so subgroups of these act too. For example $\left(g_{1}, g_{2}\right) \cdot\left(x_{1}, x_{2}\right)=\left(g_{2} x_{1}, g_{1} x_{2}\right)$. The orbits and fixed points of these actions provides the above invariants. The following result about orbits of group action is useful.

PROPOSITION 3.1. If $G$ acts on $x$ and $G_{0} \subset G$ and $h$ in normalizer of $G_{0}$, then $G_{0}(\mathrm{hx})=\mathrm{h} \cdot G_{0}(\mathrm{x})$

Proof. $g \cdot h x=h \cdot h^{-1} g h \cdot x \quad h \cdot g x=h g h^{-1} \cdot h x$. 
COROLLARY 3.3. If $\left(g_{1}, g_{2}\right)$ in $I_{f^{\prime}}$ then $I_{f}\left(g_{2} x, g_{1} y\right)=\left\{\left(g_{2} x^{\prime}, g_{1} y^{\prime}\right) \mid\left(x^{\prime}, y^{\prime}\right)\right.$ in $\left.I_{f}(x, y)\right\}$

PROPOSITION 3.4. If $(x, y) \in$ graph of $f$ then $I_{f}(x, y) \subset$ graph of $f$.

Proof. $y=f(x)$. Take $\left(g_{1}, g_{2}\right)$ in $I_{f}$. Consider $\left(g_{2}(x), g_{1}(y)\right)$. Now $g_{1} \mathrm{fg}_{2}^{-1}=f$, so $f g_{2}=g_{1} f . \quad g_{1}(y)=g_{1} f(x)=f g_{2}(x)$ so considered point is in graph of $f$.

\section{SET CASE}

These invariant subsets are easy to describe in the set case. Additionally since the invariant groups in the continuous case are subsets of the invariant groups in the set case, the partition of orbits in the continuous case is a refinement of those in the set case.

Generators of the invariant groups can be explicitly described.

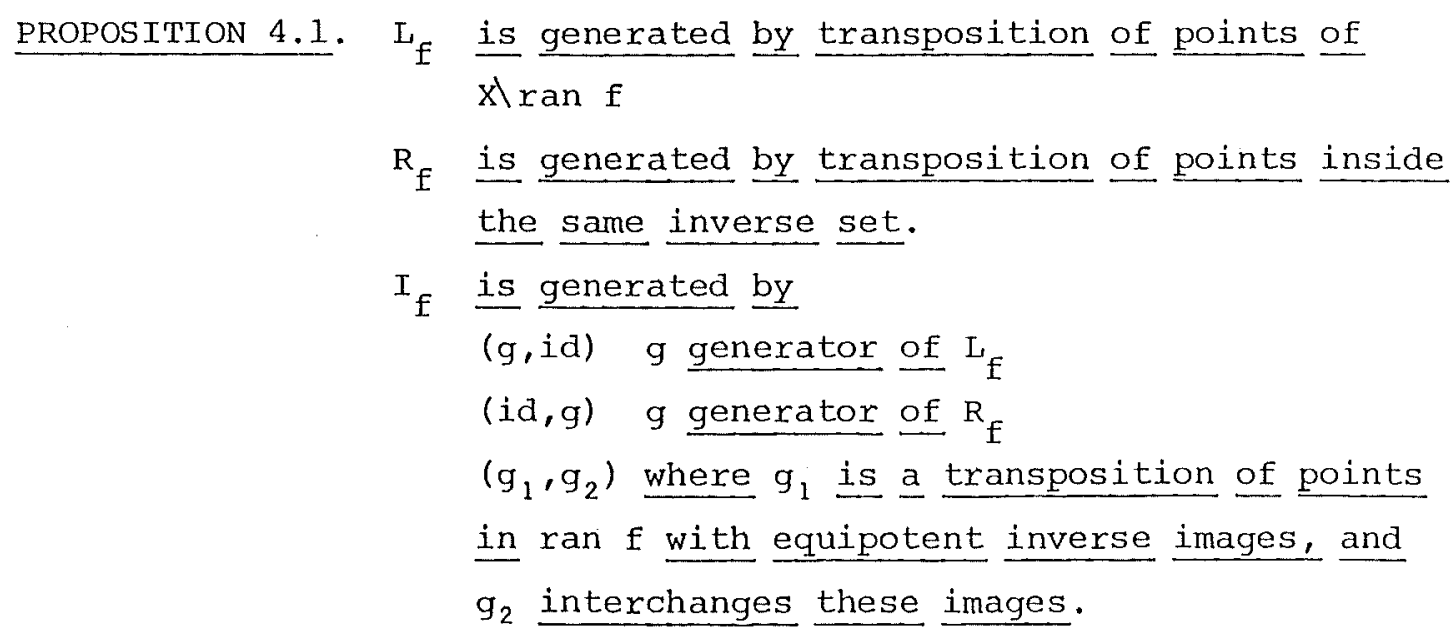

Using this one gets:

PROPOSITION 4.2 .

$$
\begin{aligned}
& \mathrm{L}_{\mathrm{f}}(\mathrm{x})=\{\mathrm{x}\} \text { iff } \mathrm{x} \text { in } \operatorname{ran} \mathrm{f} \text { or }\{\mathrm{x}\}=\mathrm{x} \backslash \operatorname{ran} \mathrm{f} \\
& K\left(L_{f}\right) \quad=\left\{\begin{array}{r}
\operatorname{ran} f \\
x
\end{array} \quad \text { if } \quad x \backslash r a n f \frac{\text { not }}{\text { a point }}\right. \\
& R_{f}(x) \quad=f^{-1} f(x) \text {. } \\
& I_{f}(x, f(x))=\text { the maximal part of the graph of } f \text { containing }(x, f(x)) \\
& \text { with all inverse images equipotent. } \\
& I_{E}(x, y) \text { for } y \neq f(x) \text { but } y \in \text { ran } f \text { equals } \\
& \operatorname{LDf}(x) \times \operatorname{LRf}(y) \backslash I_{f}(x, f(x)) \text { where } \operatorname{LDf}(x) \text {, the like domain } \\
& =\left\{x^{\prime} \mid \operatorname{card} f^{-1} f\left(x^{\prime}\right)=\operatorname{card} f^{-1} f(x)\right\} \text { and } \operatorname{LRf}(y) \text {, the like }
\end{aligned}
$$

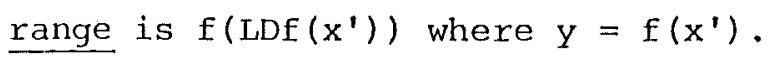

$$
\begin{aligned}
& I_{f}(x, y) \text { for } y \notin \operatorname{ran} f \text { equals } \operatorname{LDf}(x) \times X \operatorname{ran} f .
\end{aligned}
$$


Looking at all orbits of $I_{f^{\prime}}$ it is possible to recognize some orbits that necessarily belong to the graph of $f$. We say an orbit of $I_{f}$ is recognizably graph like if it looks like a graph and has a range of more than 3 points.

PROPOSITION 4.3. I $\mathrm{f}_{\mathrm{f}}(\mathrm{x}, \mathrm{y})$ is recognizably graph like iff $y=f(x)$ and $\operatorname{card} \operatorname{LRf}(y) \geqslant 3$

Let the reconstructable domain of $f, \operatorname{RDf}$, be $\{x \mid$ card $\operatorname{LRf}(f(x)) \geqslant 3\}$. It is possible to reconstruct the graph of $f$ over this set, hence PROPOSITION 4.4. $\operatorname{RDf}=\operatorname{RD} \phi f$ and $\mathrm{f}$ and $\phi \mathrm{f}$ agree on this set.

Proof. The orbits of $I_{f}$ are invariant and the graph over RDf can be reconstructed since at each point $x$ of RDf only one $y$ has $(x, y)$ with orbit like a non-constant graph.

\section{TOPOLOGICAL CASE}

Let $\mathrm{X}$ be a compact manifold, the dimension being $\geqslant 1$ if without boundary and $\geqslant 2$ if with boundary. $\quad S$ is a semigroup of continuous functions containing $G$. Although the orbits of the invariants are finer than in the set case, continuous functions are simpler than arbitxary functions. (e.g. determined on a dense set). Technical problems associated with dim $=1$, relate to the fact that then the boundary is not locally homogeneous.

PROPERTIES AND USES OF $\mathrm{L}_{\mathrm{F}}$

PROPOSITION 5.1. $K\left(L_{f}\right)=\operatorname{ran} \mathrm{f}$

Proof. Given y \& ran f. $\exists \mathrm{N}$ open $\exists \mathrm{y} . \quad \exists$ g homeomorphism supported in $N$ which moves $y$. Now $g \in L_{f}$ so $y \notin K\left(L_{f}\right)$.

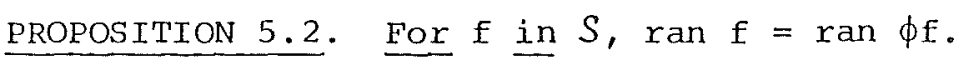
DEFINITION 5.3. $A \subset X$ is a range set if $\exists \mathrm{s} \in S \mathrm{~A}=$ ran $\mathrm{s}$. DEFINITION 5.4. $\forall$ range set $A, \forall f$ in $S, f(A)=\phi f(A)$.

Proof. Let $s \in S$ with $A=\operatorname{ran} s . \quad f(A)=f(\operatorname{ran} s)=\operatorname{ran}(f \circ s)$ $=\operatorname{ran}(\phi \mathrm{f} \circ \phi \mathrm{S})=\phi \mathrm{f}(\operatorname{ran} \phi \mathrm{s})=\phi f(\mathrm{~A})$.

The following are immediate Corollaries.

PROPOSITION 5.5. $\phi f(x) \in \cap f(A)$

$A$ a range $\operatorname{set} \exists x$

PROPOSITION 5.6. If $\mathrm{f}$ is $1-1, \phi \mathrm{f}(\mathrm{x}) \in \mathrm{f}(\cap \mathrm{A})$ 


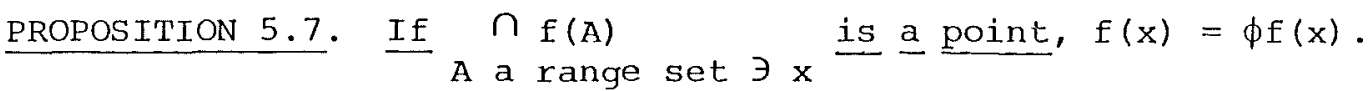

Note: If containing $\mathrm{x}$ there are arbitxarily small range sets in the metric sense, the uniform continuity of $f$ guarantees the intersection will be a point.

PROPOSITION 5.8. $\phi \mathrm{f}(\mathrm{x}) \in\left\{\mathrm{y} \mid \mathrm{f}^{-1}(\mathrm{y})\right.$ meets every range set containing $\mathrm{x}\}$.

Proof. Suppose $\exists$ range set $A \exists x$ and $\mathrm{f}^{-1} \phi f(x) \cap A=\phi$. So $\phi f(x) \& f(A)=\phi f(A)$ !

Certain conditions ensure that $S$ has the inner automorphism property.

THEOREM 5.9. If $S \subset G \cup K$, where $K$ is the semigroup of constant maps, then $S$ has $\underline{\text { i.a.p. }}$.

THEOREM 5.10. If $G \cup K^{\prime} \subset S$, where $K^{\prime}$ is the semigroup of constant maps into int $x$, then $S$ has i.a.p.

THEOREM 5.11. If $\forall$ nbd $U \exists$ range set inside $U$, then $S$ has $\underline{\text { i.a.p. }}$.

Proof. Take $x \in$ int $x$ and $N$ open ball $\exists x$. There is range set $A \subset N, A=s(X) . \quad \exists$ homeomorphism $g$ supported in $N$ so $x \in g(A)=$ $g s(x)$. By Proposition $5.7 \phi f(x)=f(x)$. Since int $x$ is dense $\phi \mathrm{f}=\mathrm{f}$.

COROLLARY 5.12. For $\mathrm{X}=S^{\mathrm{n}} \mathrm{n} \geqslant 1$ or $I^{\mathrm{n}} \mathrm{n} \geqslant 2$. If $S$ has a map into int $x$, then $S$ has i.a.p.

PROPERTIES AND USES OF $\mathrm{R}_{f}$

$R_{f}(x) \subset f^{-1} f(x)$ and the containment is usually proper. Although manifolds are nice with respect to homeomorphisms (e.g. homogeneity), inverse sets of continuous functions need not be so nice, (e.g. could have rigid points as the triod). Members of $\mathrm{R}_{\mathrm{f}}$, restricted to inverse sets, are homeomorphisms of them, so $R_{f}(x)$ could be as small as $\{x\}$ if $x$ is rigid in the space $f^{-1} f(x)$. Also, even though $f^{-1} f(x)$ is a nice topological space, it may sit thinly in $x$ and be surrounded by smaller members of the inverse partition of $f$. When $\mathrm{f}^{-1} \mathrm{f}(\mathrm{x})$ contains an open set, $\mathrm{R}_{f}(\mathrm{x})$ provides useful information. DEFINITION 5.13. $\mathrm{C}_{\mathrm{f}}=\mathrm{U}$ \{int $\left.\mathrm{f}^{-1} \mathrm{f}(\mathrm{x})\right\}$, the constancy of $\mathrm{f} . \quad \mathrm{x}$ in $\mathrm{C}_{\mathrm{f}}$ is called a point of constancy of $f . v_{f}=x C_{f}$ is the variability 
of $f$, its members are called points of variability of $f . \quad C \subset x$ is a constancy set if $\exists \mathrm{s} \in S \mathrm{C}=\mathrm{C}_{\mathrm{S}}$.

PROPOSITION 5.14. $\mathrm{C}_{\mathrm{f}}$ is open. At any $\mathrm{x} \in \mathrm{C}_{\mathrm{f}}$, thexe is an open ball $N \ni \times$ with $\mathrm{f}_{\mathrm{N}}$ constant. If $\mathrm{U}$ is a connected open set in $\mathrm{C}_{\mathrm{F}^{\prime}}$ then ${ }^{\mathrm{f}} \mathrm{u}$ is constant.

PROPOSITION 5.15. $g \in G, \mathrm{C}_{\mathrm{fg}}=\mathrm{g}^{-1} \mathrm{C}_{\mathrm{E}}$.

Proof. $\quad C_{f g}=\underset{x}{U}\left\{\right.$ int $\left.(f g)^{-1}(f g)(x)\right\}=\underset{y}{U}\left\{\right.$ int $\left.g^{-1} f^{-1} f(y)\right\}=g^{-1} C_{f}$.

PROPOSITION 5.16. int $\mathrm{x} \cap \mathrm{C}_{\mathrm{f}}=\underset{\mathrm{x}}{U}\left\{\right.$ int $\left.\mathrm{R}_{\mathrm{f}}(\mathrm{x})\right\}$

Proof. Take $x \in$ int $x \cap C_{f^{*}} \quad \exists$ open ball $N \exists x_{\text {with }} f_{\mid N}$ constant. $\forall x^{\prime} \in N \exists g$ supported in $N, g(x)=x^{\prime} . g \in R_{f^{\prime}}$ so $\mathrm{N} \subset \mathrm{R}_{\mathrm{f}}(\mathrm{x})$, and $\mathrm{x} \in$ int $\mathrm{R}_{\mathrm{f}}(\mathrm{x})$. For the reverse containment take $\mathrm{x}^{\prime} \in$ int $\mathrm{R}_{\mathrm{f}}(\mathrm{x})$. Since the orbit of any boundary point stays on the boundary, $x$ must belong to int $x$, so $x^{\prime} \epsilon$ int $x$. Now $\exists$ open ball $N \ni x^{\prime}$ with $N \subset R_{f}(x)$. Take any $x^{\prime \prime} \in N, \exists g$ in $R_{f} g(x)=x^{\prime \prime}$. $f\left(x^{\prime \prime}\right)=f g(x)=f(x)$, so $f_{\mid N}$ is constant, thus $x^{\prime} \in C_{f}$. PROPOSITION 5.17. $\mathrm{C}_{\mathrm{f}}=\mathrm{C}_{\phi \mathrm{f}}$.

Proof. By previous Proposition 5.16, int $x \cap C_{f}=$ int $x . \cap C_{\phi f}$. For $x \in C_{f} \cap \partial x \exists$ half-open ball $N \exists x, N \subset C_{f}$. So $f_{\mid N}$ is constant. So $\mathrm{N} \cap$ int $\mathrm{x} \subset \mathrm{C}_{\phi \mathrm{f}^{\prime}}$, thus $\phi \mathrm{f} \mid(\mathrm{N} \cap$ int $\mathrm{x})$ is constant and by continuity $\phi \mathrm{f}_{\mathrm{N}}$ is constant, so $\mathrm{x} \in \mathrm{C}_{\phi \mathrm{f}}$.

PROPOSITION 5.18. $\mathrm{N}$ open and connected. ${ }^{\mathrm{f}} / \mathrm{N}$ is constant iff $\phi \mathrm{f} / \mathrm{N}$ is. PROPOSITION 5.19. For X connected. If $\mathrm{f}$ and $\phi \mathrm{f}$ agree on $V_{\mathrm{f}}\left(=\mathrm{V}_{\phi \mathrm{f}}\right)$, then $\mathrm{f}=\phi \mathrm{f}$.

Proof. Denote the components of $\mathrm{C}_{\mathrm{f}}$ by $\mathrm{C}_{\mathrm{f}}^{\alpha}$. These are open connected subsets of $\mathrm{X}$ and $\mathrm{f}_{\mathrm{C}_{\mathrm{f}}^{\alpha}}$ is constant. Case 1 . One $\mathrm{C}_{\mathrm{f}}^{\alpha}$ is closed, hence equals $x$ and $f^{f}$ is a constant. So $f=\phi f$. Case 2, each $C_{f}^{\alpha}$ has limit point $x_{\alpha} \& C_{f}^{\alpha}$. Now $x_{\alpha} \& C_{f}^{\beta} \beta \neq \alpha$ since $\mathrm{C}_{\mathrm{f}}^{\beta}$ are open and disjoint from $\mathrm{C}_{\mathrm{f}}^{\alpha}$. So $\forall \alpha, \mathrm{x}_{\alpha} \in \mathrm{V}_{\mathrm{f}}$ where $\mathrm{f}\left(\mathrm{x}_{\alpha}\right)=$ $\phi \mathrm{f}\left(\mathrm{x}_{\alpha}\right)$. By continuity of $\mathrm{f}$ and $\phi \mathrm{f}, \mathrm{f}\left(\mathrm{x}_{\alpha}\right)=\mathrm{f}\left(\mathrm{C}_{\mathrm{f}}^{\alpha}\right), \phi \mathrm{f}\left(\mathrm{x}_{\alpha}\right)=\phi \mathrm{f}\left(\mathrm{C}_{\mathrm{f}}^{\alpha}\right)$, so $\left.\right|_{C_{f}} ^{\alpha}=\left.\phi f\right|_{C_{f}} ^{\alpha} \forall \alpha . \quad f=\phi f$.

Constancy sets are nearly dual to range sets. The analogue to proposition 5.4 is: 
PROPOSITION 5.20. $\forall x \in v_{f} \forall$ constancy set $C$.

If $f(x) \in C$, then $f(x) \in C \cup \partial x$.

Proof. Suppose $\phi f(x) \in$ int $x$ and $\exists s \in S$ with $f(x) \in C_{s}$ and $\phi f(x) \& C_{S} \cdot \exists$ open ball $v_{1} \ni \phi f(x)$, open (or half-open) ball $v_{2} \exists \mathrm{f}(\mathrm{x})$, U open $\exists \mathrm{x}$ such that $\mathrm{v}_{1} \cap \mathrm{v}_{2}=\varnothing, \mathrm{f}(\mathrm{U}) \subset \mathrm{v}_{2}, \phi \mathrm{f}(\mathrm{U}) \subset \mathrm{v}_{1}$, and $s \mid v_{2}$ is constant. Now $\exists x^{\prime}$ in $U \phi f(x) \neq \phi f\left(x^{\prime}\right)$ since $x \in v_{\phi f}=v_{f}$. Also $\exists y^{\prime} \in v_{1} \phi s(\phi f(x)) \neq \phi s\left(y^{\prime}\right)$ since $\phi f(x) \& C_{\phi s} \cdot \exists g$ a homeomorphism supported in $V_{1}, g$ fixed at $\phi f(x)$ and $g\left(\phi f\left(x^{\prime}\right)\right)=y^{\prime}$. Consider sgf. $\left.{ }^{s g f}\right|_{U}$ is constant while $\left.\phi(s g f)\right|_{U}$ is not: So $\phi f(x) \in C_{S}$. PROPOSITION 5.21. For $x \in V_{f^{\prime}} \phi f(x) \in \underset{f(x) \in C \text { a constancy set }}{\cap} \cup$ For manifolds without boundary, constancy sets play a truly dual role to range sets.

PROPOSITION 5.22. For $\mathrm{X}$ a manifold without boundary, $\mathrm{x} \in \mathrm{V}_{f}, \mathrm{C}$ a constancy set, $f(x) \in C$ iff $\phi f(x) \in C$.

Proof. Use $\phi^{-1}$ for other direction.

PROPOSITION 5.23. $\mathrm{x}$ a manifold without boundary, $\mathrm{C}$ constancy set $v_{f} \cap f^{-1}(C)=v_{f} \cap \phi f^{-1}(C)$.

THEOREM 5.24. $X$ a connected manifold without boundary. If $S$ has a map with non-empty constancy, then $S$ has i.a.p.

Proof. Let $s \in S$ with $\mathrm{C}_{\mathrm{s}} \neq \varnothing$. If $\mathrm{C}_{\mathrm{s}}=\mathrm{x}$, then $\mathrm{s}$ is constant, and $S$ contains all constants, so has i.a.p. If $\exists x^{\prime} \notin C_{S}$. Take any $f \in S$ and $x \in v_{f}$, suppose $\phi f(x) \neq f(x) . \quad \exists g \in G g f(x) \in C_{S}$ and $g \phi f(x) \& C_{S}$. Consider the constancy set $C_{s g}=g^{-1} C_{S} \cdot f(x) \in C_{s g}$, but $\phi f(x) \notin C_{s g} ! \quad$ So $f(x)=\phi f(x)$ for all $x \in V_{f} \cdot \quad$ So $f=\phi f$.

THEOREM 5.25. $X$ connected manifold without boundary. If $S$ contains $E$, the semigroup of all onto maps, then $S$ has i.a.p.

PROPERTIES AND USES OF $I_{\mathrm{f}}$

As in the set case, this relates to the graph of $f$. Certainly $\left(g_{1}, g_{2}\right) \in I_{f}$ if it belongs to $L_{f} \times R_{f}$. More generally, $\left(g_{1}, g_{2}\right) \in I_{f}$ means that $g_{2}$ moves topologicall $l_{Y}$ equivalent inverse images that sit in the surrounding partition in the same way, while $g_{1}$ moves the corresponding images. The analogue to proposition 4.3 is given and applies to those $\mathrm{x}$ with $\mathrm{f}$ particularly nice near $\mathrm{x}$. 
DEEINITION 5.26. $f$ is reconstructable at $x$ if $x \in$ int $x \cap v_{f}$ and $\exists \mathrm{y}_{0} \forall \mathrm{y} \neq \mathrm{y}_{0} \exists \mathrm{U}$ open ball $\ni \mathrm{x}$ with $\mathrm{I}_{\mathrm{f}}(\mathrm{x}, \mathrm{y}) \supset \mathrm{U} \mathrm{x}\{\mathrm{y}\}$.

PROPOSITION 5.27. If $\mathrm{f}$ is reconstructable at $\mathrm{x}$ and $\mathrm{y}_{0}$ as in the definition then $f(x)=y_{0}$ and $\exists$ U open $\exists x$ with $I_{f}\left(x, y_{0}\right)$ a graph piece with domain part. $\supset U$.

Proof. For $y \neq y_{a} \exists U_{y}$ open $\ni x I_{f}(x, y) \supset U_{Y} x\{y\}$. Now as $x \in V_{f^{\prime}} f$ is not constant over any nbd of $x$, so $I_{f}(x, y)$ cannot be a piece of the graph of $f$ over $x$. The only orbit that can be a piece of the graph is $I_{f}\left(x, y_{0}\right)$, so $f(x)=y_{0}$. Let $U=$ the union of all $u_{y}^{\prime} ' s$. Clearly given any $x^{\prime} \in \cup \exists\left(g_{1}, g_{2}\right) \in I_{f} g_{2}(x)=x^{\prime}$, so $U \subset$ domain part of $I_{f}(x, f(x))$.

PROPOSITION 5.28. If $\mathrm{f}$ is reconstructable at $\mathrm{x}$, and $\mathrm{g}^{\prime}, \mathrm{g} \in G$, then $g^{\prime} f g$ is reconstructable at $g^{-1} \mathrm{x}$.

Proof. $I_{g^{\prime} f g}=\left(g^{\prime}, g^{-1}\right) I_{g}\left(g^{\prime}, g^{-1}\right)^{-1}$. For $y \neq g^{\prime} f(x)$, $I_{g^{\prime} f g}\left(g^{-1} x, y\right)=\left(g^{\prime}, g^{-1}\right) I_{f}\left(x, g^{\prime^{-1}} y\right)$ which contains a set of the form $U x\{y\}, U \ni g^{-1} x$.

PROPOSITION 5.29. $\mathrm{f}$ is reconstructable at $\mathrm{x}$ iff $\phi \mathrm{f}$ is, and $\exists \mathrm{U}$ open $\exists \times$ such that $\mathrm{f}_{\mid \mathrm{U}}=\phi \mathrm{f}_{\mathrm{U}}$.

PROPOSITION 5.30. If $f$ is reconstructable at $x,\left(g_{1}, g_{2}\right) \in I_{f^{\prime}}$ and $x^{\prime}=g_{2}(x)$, then $f$ is reconstructable at $x^{\prime}$.

Proof. $f=g_{1} \mathrm{fg}_{2}^{-1}$, which is reconstructable at $g_{2} \mathrm{x}=\mathrm{x}^{\prime}$, by Proposition 5.28 .

DEFINITION 5.31. The reconstructable domain of $f, \cdot R D=\{x \mid f$ is reconstructable at $\mathrm{x}\}$.

PROPOSITION 5.32. If $\mathrm{f}$ is reconstructable at $\mathrm{x}$, then the domain part of $I_{f}(x, f(x))$ is an open subset of RDf.

Proof. Let $x^{\prime} \in$ domain part of $I_{f}(x, f(x))$. So $\left(x^{\prime}, f\left(x^{\prime}\right)\right) \in$ $I_{f}(x, f(x))$ which means $I_{f}(x, f(x))=I_{f}\left(x^{\prime}, f\left(x^{\prime}\right)\right)$, so $\exists\left(g_{1}, g_{2}\right) \in I_{f}$ with $x^{\prime}=g_{2}(x)$, so $f$ is reconstructable at $x^{\prime}$. By Proposition 5.27 $\exists U^{\prime}$ open $\exists x^{\prime}$ with the domain part of $I_{f}\left(x^{\prime}, f\left(x^{\prime}\right)\right) \supset U^{\prime}$, so $U^{\prime} \subset$ the domain part of $I_{f}(x, f(x))$. So the domain part of $I_{f}(x, f(x))$ is open. 
The following three propositions are corollaries. PROPOSITION 5.33. RDf is open.

PROPOSITION 5.34. If RDf is dense, then $\mathrm{E}=\phi \mathrm{f}$. PROPOSITION 5.35. If $X$ is connected and RDf is dense in $\mathrm{V}_{\mathrm{E}^{\prime}}$ then $\mathrm{f}=\phi \mathrm{f}$.

Many functions have RDf dense. The following propositions give some necessary conditions.

DEFINITION 5.36. For $V, U$ open $X$, let $I_{f}[V, U]=\left\{\left(g_{1}, g_{2}\right) \in I_{f} \mid g_{1}\right.$ is supported in $\mathrm{V}, \mathrm{g}_{2}$ is supported in $\mathrm{U}$.

DEEINITION 5.37. $f$ is movable at $x$ if $x \in$ int $x \cap v_{f}$ and $\forall$ open $V \ni f(x)$, the domain part of $I_{f}\left[V, f^{-1}(V)\right](x, f(x))$ contains a neighbourhood of $x$.

PROPOSITION 5.38. If $f$ is movable at $x$, then $I_{f}\left(x^{\prime}, f(x)\right)$ is not graphlike when $f\left(x^{\prime}\right) \neq f(x)$.

Proof. Choose V $\ni \mathrm{f}(\mathrm{x})$ with $\mathrm{f}\left(\mathrm{x}^{\prime}\right) \& \mathrm{~V}$. Let $\mathrm{U}$ open $\ni \mathrm{x}$ be in the domain part of $I_{f}\left[V, f^{-1}(V)\right](x, f(x))$. $f(U)$ consists of more than one point as $x \in V_{f} \cdot \quad$ Now $I_{f}\left(x^{\prime}, f(x)\right) \supset I_{f}\left[V, f^{-1}(V)\right]\left(x^{\prime}, f(x)\right) \supset\left\{x^{\prime}\right\} x f(U)$.

PROPOSITION 5.39. $f$ is movable at $x$ iff $\phi f$ is.

PROPOSITION 5.40. If $\mathrm{f}$ is movable at $\mathrm{x}$, then $\mathrm{f}$ is reconstructable at $x$.

Proof. Let $y_{0}=f(x)$. Let $y \neq y_{0}$, choose $V$ open $\exists f(x)$ with $y \& V$. Let $U$ be open containing $x$ in the domain part of $I_{f}\left[V, f^{-1}(V)\right]$ $(x, f(x)) . \quad I_{f}(x, y) \supset I_{f}\left[V, f^{-1}(V)\right](x, y) \supset U \times\{y\}$. So $f$ is reconstructable at $x$.

DEFINITION 5.41. $f$ is a local covering at $x$ if $\exists$ open (or half-open) bal1 $U_{1} \in \times$ with $f\left(U_{1}\right)$ open and $f^{-1} f\left(U_{1}\right)=U_{1} \cup U_{2} \cup \ldots U U_{k}$ a finite disjoint union of open sets and $\left.f\right|_{U_{i}}$ a homeomorphism.

PROPOSITION 5.42. If $x \in$ int $x$ and $f$ is a local covering at $x$, then $\mathrm{f}$ is movable at $\mathrm{x}$.

Proof. For $V$ open $\ni f(x)$ take $U_{1}$ an open ball about $x$ with $\mathrm{f}\left(\mathrm{U}_{1}\right) \subset \mathrm{V}$ and $\mathrm{f}^{-1} \mathrm{E}\left(\mathrm{U}_{1}\right)=U_{1} U \ldots U \mathrm{U}_{k^{\prime}}$ disjoint open balls, $\left.\mathrm{f}\right|_{U_{i}} a$ homeomorphism. Take $\mathrm{N}$ open ball $\exists \mathrm{x}$ with $\mathrm{cl}(\mathrm{N}) \subset \mathrm{U}_{1}$. Take any $\mathrm{x}^{\prime}$ in $\mathrm{N}$, let $\mathrm{g}$ be homeomorphism of $\mathrm{U}_{1}$ supported in $\mathrm{N}$ taking $\mathrm{x}$ to $\mathrm{x}^{\prime}$, 
extend to $g_{2}$ a homeomorphism of $x$ by $g_{2} \mid u_{i}=\left(f_{\mid u_{i}}\right)^{-1} f g\left(\left.f\right|_{u_{i}}\right)^{-1} f$, and id elsewhere.

Let $g_{1} \mid f\left(U_{1}\right)=f g\left(f U_{U_{1}}\right)^{-1}$, and id elsewhere. $\left(g_{1}, g_{2}\right)$ is in $I_{f}\left[V, f^{-1}(V)\right]$ $g_{2}(x)=x^{\prime}$, so the domain part contains $N$, so $f$ is movable at $x$.

PROPOSITION 5.43. If $\mathrm{f}$ is a local covering at $\mathrm{x}$, then $\phi \mathrm{f}$ is a local covering of $f$, and $f=\phi f$ in a neighbourhood of $x$.

Proof. Let $\mathrm{N}$ be open or half-open $\exists \mathrm{x}$, where $\mathrm{f}$ is a homeomorphism on each component of $\mathrm{f}^{-1} \mathrm{f}(\mathrm{N})$. For each point of $\mathrm{N} \cap$ int $\mathrm{X}$, $\mathrm{f}$ is movable, so $\mathrm{f}_{\mid(\mathrm{N} \cap \text { int } \mathrm{X})}=\phi \mathrm{f}_{\mid(\mathrm{N} \cap \text { int } \mathrm{X})} \cdot \quad$ By continuity $\mathrm{f}_{\mid \mathrm{N}}=\left.\phi \mathrm{f}\right|_{\mathrm{N}}$. Take $x^{\prime} \in N, \quad f^{-1} f\left(x^{\prime}\right)=(\phi f)^{-1}(\phi f)\left(x^{\prime}\right)$ as follows. Take $x^{\prime \prime}$ in $f^{-1} f\left(x^{\prime}\right), f$ is a local covering there so $\phi f\left(x^{\prime \prime}\right)=f\left(x^{\prime \prime}\right)=f\left(x^{\prime}\right)=$ $\phi f\left(x^{\prime}\right)$ thus $x^{\prime \prime} \in(\phi f)^{-1}(\phi f)\left(x^{\prime}\right)$. To show containment in the other direction. Let $x^{\prime \prime} \in(\phi f)^{-1}(\phi f)\left(x^{\prime}\right)$. Now if $x^{\prime \prime} \& f^{-1} f\left(x^{\prime}\right)$, $f\left(x^{\prime}\right) \neq f\left(x^{\prime \prime}\right)$, and $I_{f}\left(x^{\prime \prime}, f\left(x^{\prime}\right)\right)$ is not graph-like by Proposition 5.38 but this equals $I_{f}\left(x^{\prime \prime}, \phi f\left(x^{\prime \prime}\right)\right)$ which is part of the graph of $\phi f !$ So $\mathrm{f}^{-1} \mathrm{E}(\mathrm{N})=(\phi f)^{-1}(\phi E)(N)$ and $\mathrm{f}$ and $\phi E$ agree there. Therefore $\phi E$ is a local covering at $x$.

Immediately one gets

PROPOSITION 5.44. If $\mathrm{f}$ is a local covering on a dense set, then $\phi \mathrm{f}=\mathrm{f}$.

PROPOSITION 5.45. $X$ connected and $f$ is a local covering on a dense subset of $V_{f}$, then $\phi \mathrm{f}=\mathrm{f}$.

THEOREM 5.46. If $S$ is semigroup with all $f \in S$ local coverings on a dense set, $S$ has the i.a.p.

THEOREM 5.47. If $S$ is semigroup and $X$ connected and all $f \in S$ are local coverings on a dense set of $v_{f}$, then $S$ has i.a.p.

THEOREM 5..48. $S=M$, the semigroup of all 1-1 maps, has i.a.p.

The results using constancy sets are refined for manifolds with boundary.

DEFINITION 5.49. The boundary variability of $\mathrm{E}, \mathrm{BV}_{\mathrm{f}}=\{\mathrm{x} \in \partial \mathrm{x} \mid \forall \mathrm{U}$ open $\ni x, f \mid u \cap \partial x$ is not constant and $\exists$ half-open $N \exists x$ such that $f$ is reconstructable at each point of $\mathrm{N} \cap$ int $\mathrm{x}$ \}.

PROPOSITION 5.50. For $\mathrm{g} \in G, \mathrm{BV}_{\mathrm{sg}}=\mathrm{g}^{-1} \mathrm{BV}_{\mathrm{S}}$. 
PROPOSITION 5.51. $\quad \mathrm{BV}_{\mathrm{E}}=\mathrm{BV}_{\phi \mathrm{E}}$.

PROPOSITION 5.52. For $x \in \mathrm{V}_{\mathrm{f}}$ and $\mathrm{C}_{\mathrm{s}}$, a constancy set. If $\mathrm{f}(\mathrm{x}) \in \mathrm{C}_{\mathrm{s}}$ then $\phi f(x) \in C_{S} U \partial x \backslash_{B V}$.

Proof. By Theorem $5.20 \phi f(x) \in \mathrm{C}_{\mathrm{S}} \cup \partial \mathrm{x}$. The proof of Theorem 5.20 applies here with very little modification. The only case to be eliminated is $\phi \mathrm{f}(\mathrm{x}) \in \mathrm{BV}_{\mathrm{s}}$. The proof of 5.20 applies with the following additional conditions. Let $v_{1}$ be half-open ball $\exists \phi E(x)$. Choose $y^{\prime} \in v_{1}$ to be in int $x$ or $\partial x$, respectively when $\phi f\left(x^{\prime}\right)$ is in int $x$ or $\partial x$.

PROPOSITION 5.53. Given s with $\partial x \backslash B V_{S} \subset C_{S}$; For $x \in v_{f^{\prime}} f(x) \in C_{S}$ iff $\phi f(x) \in C_{S} \cdot \quad V_{f} \cap f^{-1}\left(C_{S}\right)=V_{f} \cap(\phi f)^{-1}\left(C_{S}\right)$.

THEOREM 5.54. $x$ is connected manifold with boundary. If for each Component of $x$, there is $s \in S$ with $C_{S}$ meeting it and $B V_{s}$ meeting all components, then $S$ has $\underline{\text { i.a.p. }}$.

Proof. Take any $f \in S$ and $x \in V_{f}$. Let $y \neq f(x)$. If $f(x) \in \partial x$, take $s$ so $C_{S}$ meets the component containing $f(x)$ and $B V_{S}$ meeting all components. If $f(x) \in$ int $x$, take s satisfying only the second part.

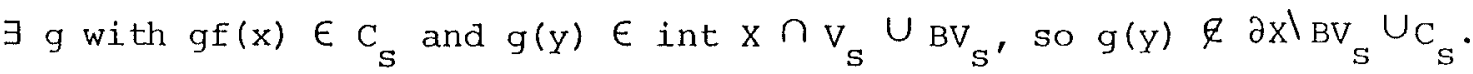
Now $f(x) \in C_{S_{g}}$ so $y \notin C_{s g} U \partial x \backslash_{B V}{ }_{s g^{\prime}}$ so $\phi f(x) \neq y$. Thus $\phi f(x)=f(x)$ for each $x \in V_{f}$, and $\phi f=f$.

THEOREM 5.55. For X connected. If $S$ contains $E$, the semigroup of all onto maps, then $S$ has i.a.p.

\section{GREENS RELATIONS}

Greens relations partition the semigroup into collections of elements that generate like ideals. The group invariants here partition $S$ similarly. Two elements will be related if they have the same group invariant.

THEOREM 6.1 . If $f \mathcal{L}_{f^{\prime}}$, then $R_{f}=R_{f^{\prime}}$. If $f R_{f^{\prime}}$, then $L_{f}=L_{f^{\prime}} \cdot$ Proof. $f \mathcal{L}_{f^{\prime}}$ so $\exists k, k^{\prime} \in S$ with $f=k f^{\prime}, f^{\prime}=k^{\prime} f$. Take $g \in R_{f}$.

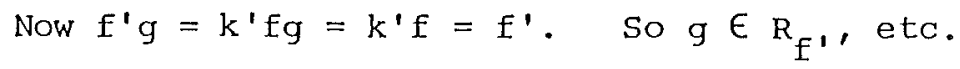

Converse results hold in special circumstances.

THEOREM 6.2. For the full semigroup of set transformations.

$$
f^{\prime} f^{\prime} \quad \text { iff } R_{f}=R_{f} \text { ' }
$$


When card $\mathrm{x} \backslash$ ran $\mathrm{f} \geqslant 2$ and cंard: $\mathrm{x} \backslash \operatorname{ran} \mathrm{f}^{\prime} \geqslant 2$

$$
\mathrm{L}_{\mathrm{F}}=\mathrm{L}_{\mathrm{f}^{\prime}} \quad \underline{\text { imply }} \quad \mathrm{f} \mathbb{R}_{\mathrm{E}^{\prime}} \text {. }
$$

Proof. Use Proposition 4.2 , and the characterization of $\mathbb{R}$ and $\mathcal{L}$ by like partitions and ranges respectively [ 1$]$.

THEOREM 6.3. For $S(X)$ the full semigroup of continuous maps for regular elements of $S(X), L_{f}=L_{F^{\prime}}$ implies $f R_{f^{\prime}}$.

Proof. Magil1 and Subbiah [8] showed $R$ was characterized by 1ike ranges.

\section{SEMIGROUPS WUTH OUTER AUTOMORPHISMS}

In light of the previous results, the candidates that possibly could have outer automorphisms must be deficient in range sets (i.e. not enough into maps) and constancy sets (i.e. not enough squashing maps). Also the semigroup must contain functions that are not nice (i.e. non-reconstructable parts of their graphs) .

Outer automorphisms on $S$ can sometimes be "inner" with respect to bijections on the set $x$. This can happen if there are any bijections that commute with all homeomorphisms. In the case presented in this paper, the Centralizer of $G$ in all bijections is trivial, so no outer automorphisms come about in this way. For $\mathrm{x}$ of dim 1 with boundary, this is a possibility. (c.f. Wood [11])

Some general observations

Some examples of semigroups with outer automorphisms are given. They are all of the form $\langle G \cup C\rangle$, with $C \subset T$ the semigroups of all continuous transformations. -Some conditions are given ensuring that any automorphism of $C$ extends to an outer automorphism of $S$.

If $S=\langle G \cup C\rangle$ is free in the sense that all words with letters alternating from $G$ and $C$ are unique, then any non-identity automorphism, $\phi$, of $\mathcal{C}$ extends to an outer automorphism of $S$, by letting it be the id on g's and $\phi$ on c's. More generally, if whenever two words are the same, the two words with the c's replaced by $\phi c^{\prime} s$ are the same, $\phi$ will extend. Theorems are given that combine reduction rule conditions making the word structure of $S$ simple, together with conditions on the group invariants that make the words essentially unique.

$\mathrm{L}_{\mathrm{C}}, \mathrm{R}_{\mathrm{C}}$, ani $\mathrm{I}_{\mathrm{C}}$ are defined for $\mathrm{C} \in \mathrm{C}$, but they need not be 
invariant under automorphisms of $\mathrm{C}$.

\section{Results}

PROPOSITION 7.1. Let $\mathcal{C}$ be a semigroup of transformations and $\phi \underline{\text { be }}$ an automorphism of $\mathcal{C}, \phi \neq$ id. If the following hold:

$$
\begin{aligned}
& \forall \mathrm{c} \in \mathrm{C} \cap \mathrm{G}, \quad \phi_{\mathrm{C}}=\mathrm{c} \\
& \forall \mathrm{c} \quad \forall \mathrm{g} \quad \text { gc } \in C \text { imply } \phi(\mathrm{gc})=\mathrm{g} \phi \mathrm{C} \\
& \forall \mathrm{c} \quad \mathrm{R}_{\mathrm{C}}=\mathrm{G} \\
& \forall \mathrm{C} \quad \mathrm{L}_{\mathrm{C}}=\mathrm{L}_{\phi \mathrm{C}}
\end{aligned}
$$

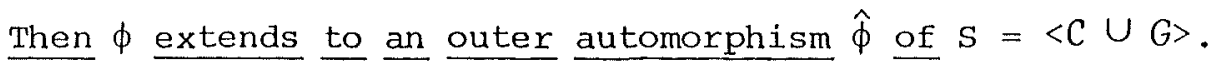

proof. $S=G \cup G C$ since $\forall g \forall C \quad c g=c . \quad$ Define $\hat{\phi}(g)=g ;$ $\hat{\phi}(g \mathrm{c})=\mathrm{g} \phi \mathrm{C} . \quad \hat{\phi}$ is well defined since, if $g=g^{\prime}, \hat{\phi}(g)=\hat{\phi}\left(g^{\prime}\right)$, if $g=g^{\prime} \mathrm{c}$, then $\mathrm{c} \in G$, so $\hat{\phi}(\mathrm{g})=\mathrm{g}=\mathrm{g}^{\prime} \mathrm{c}=\hat{\phi}\left(\mathrm{g}^{\prime} \mathrm{c}\right)$ and if $\mathrm{gc}=\mathrm{g}^{\prime} \mathrm{c}$, then $c=g^{-1} g^{\prime} c^{\prime}$, so $\phi c=g^{-1} g^{\prime} \phi c^{\prime}$ and $\hat{\phi}\left(g^{\prime} c^{\prime}\right)=g^{\prime} \phi c^{\prime}=g \phi c=\hat{\phi}(g c)$. $\hat{\phi}$ is a homomorphism from: $\hat{\phi}\left(g g^{\prime}\right)=\hat{\phi}(g) \hat{\phi}\left(g^{\prime}\right)$. $\hat{\phi}\left(g g^{\prime} \mathrm{C}\right)=g g^{\prime} \phi \mathrm{C}=\hat{\phi}(g) \hat{\phi}\left(g^{\prime} \mathrm{C}\right)$. $\hat{\phi}\left(g c g^{\prime}\right)=\hat{\phi}(g c)=g \phi c=g \phi c g^{\prime}=\hat{\phi}(g c) \hat{\phi}\left(g^{\prime}\right)$. $\hat{\phi}\left(g c g^{\prime} c^{\prime}\right)=\hat{\phi}\left(g c c^{\prime}\right)=g \phi\left(c c^{\prime}\right)=g \phi c g^{\prime} \phi c^{\prime}=\hat{\phi}(g c) \hat{\phi}\left(g^{\prime} C^{\prime}\right)$. Consider $\phi^{-1}, L_{\phi^{-1} \mathrm{C}}=\mathrm{L}_{\phi \phi^{-1} \cdot \mathrm{C}}=\mathrm{L}_{\mathrm{C}}$, so $\phi^{-1}$ extends to an inverse homomorphism. $\hat{\phi} \neq i d$, so $\hat{\phi}$ is an outer automorphism.

PROPOSITION 7.2. Same as above with second condition replaced by $\forall \mathrm{C} \forall \mathrm{g} g \mathrm{C} \in \mathrm{C}$ implies $\mathrm{g} \in \mathrm{L}_{\mathrm{C}}$.

Proof. If gc $\in C$; then $g \in L_{C}$ so $c=g c . \quad g \in L_{C}$ gives $g \in \mathrm{L}_{\phi \mathrm{C}^{\prime}}$, so $\phi(\mathrm{gc})=\phi \mathrm{c}=\mathrm{g} \phi \mathrm{C}$.

EXAMPLE 7.3. Let $x$ be a compact manifold with each component, $x_{i}$, $i=1, \mathrm{~m}$, topologically distinct. Let $K$ be a semigroup of transformations on the set $\{1,2, \ldots, m\}$. Choose points $y_{i} \in x_{i}$. There is a natural semigroup on $x$ isomorphic to $K$. Let $C=\left|c_{k}: x \rightarrow x\right| c_{k}\left(x_{i}\right)$ $=y_{k(i)} k$ in $\left.k\right\}$. Let $\phi$ be any automorphism of $C$ such that $\forall \mathrm{C} \in C$ $\operatorname{ran} \mathrm{c}=\operatorname{ran} \phi \mathrm{c}$.

Proof. Use Theorem 7.1. In fact $\mathcal{C} \cap G=\varnothing$. gc $\in \mathcal{C}$, imply $g$ is the id on $\operatorname{ran} \mathrm{c}$, so $\mathrm{gc}=\mathrm{c}$. Also $\mathrm{g} \phi \mathrm{c}=\phi \mathrm{c}$ as $\mathrm{c}$ and $\phi \mathrm{c}$ have same range.

EXAMPLE. Let $\mathrm{X}$ be the union of disk, anulus, and disk with 2 holes with two maps as shown. Their interchange is an outer automorphism. 


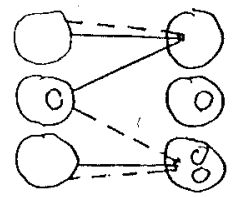

This shows connectivity of $\mathrm{X}$ is required in Theorem 5.19 , as all functions agree over $V_{E}$. Also the graph is not recognizable anywhere for these maps.

The fact that $x$ is not connected plays a strong part of the previous examples. One aspect was that the group of homeomorphism need not be globally homogeneous. It is this aspect that is important in the following examples.

PROPOSITION 7.4. Let $\mathcal{C}$ be a semigroup of transformations and $\phi$ be an automorphism of $\mathcal{C}, \phi \neq i d$. If the following hold:

$\forall \mathrm{C} \in C \cap G, \quad \phi \mathrm{C}=\mathrm{C}$

$\forall \mathrm{C} \forall \mathrm{g}, \mathrm{g}^{\prime} \quad \mathrm{gCg}^{\prime} \in \mathcal{C} \underline{\mathrm{imply}} \phi\left(\mathrm{gcg}^{\prime}\right)=g \phi \mathrm{cg}{ }^{\prime}$

$\forall \mathrm{C}, \mathrm{C}^{\prime} \forall \mathrm{g} \quad \mathrm{CgC}^{\prime}=\mathrm{gC}^{\prime}$

$\forall \mathrm{C} \quad I_{\mathrm{C}}=I_{\phi C}$

Then $\phi$ extends to an outex automorphism of $S=\langle\mathcal{C} \cup G\rangle$.

Proof. $S=G \cup G C G$ by the third condition. Define $\phi(g)=g$; $\phi\left(g_{c g}^{\prime}\right)=g_{\phi c g^{\prime}}$. Check well defined.

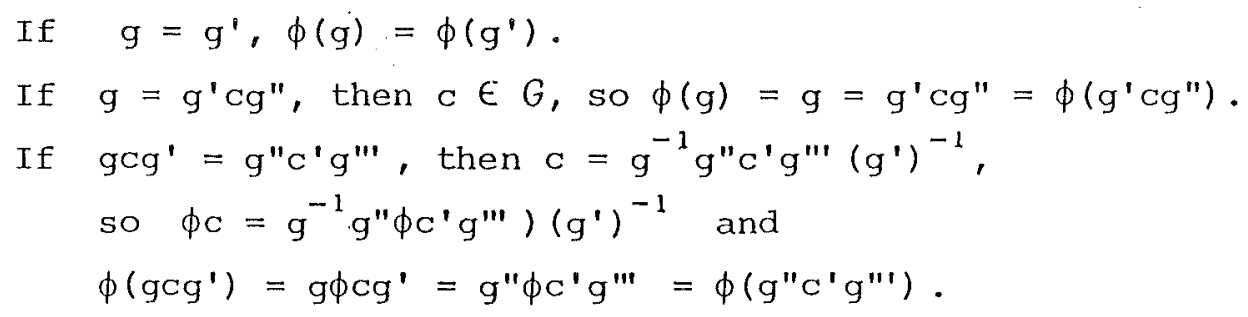

Check homomorphism.

$\phi\left(g g^{\prime}\right)=\phi(g) \phi\left(g^{\prime}\right)$.

$\phi\left(g g^{\prime} \mathrm{Cg} g^{\prime \prime}\right)=g g^{\prime} \phi \mathrm{cg} "=\phi(g) \phi\left(g^{\prime} \mathrm{Cg} "\right)$.

$\phi\left(g^{\prime} g^{\prime \prime}\right)=g \phi c g^{\prime} g^{\prime \prime}=\phi\left(g c g^{\prime}\right) \phi\left(g^{\prime \prime}\right)$.

$\phi\left(g c g^{\prime} g^{\prime \prime} c^{\prime} g^{\prime \prime \prime}\right)=\phi\left(g^{\prime} g^{\prime \prime} c^{\prime} g^{\prime \prime \prime}\right)=g g^{\prime} g^{\prime \prime} \phi c^{\prime} g^{\prime \prime \prime}=g \phi c g^{\prime} g^{\prime \prime} \phi c^{\prime} g^{\prime \prime \prime}$

$=\phi\left(g c g^{\prime}\right) \phi\left(g^{\prime \prime} c^{\prime} g^{\prime \prime \prime}\right)$.

Consider $\phi^{-1}, I_{\phi^{-1} \mathrm{C}}=\mathrm{I}_{\phi \phi^{-1} \mathrm{C}}=\mathrm{I}_{\mathrm{C}}$, so $\phi^{-1}$ extends to an inverse homomorphism. $\phi \neq i d$, so $\phi$ is an outer automorphism.

DEFINITION 7.5. $f \in T$ is a $G$-invariant retract if $f E=f$ and $g(\operatorname{ran} \mathrm{f})=\operatorname{ran} \mathrm{f} \forall \mathrm{g} \in G$.

PROPOSITION 7.6. $\quad \underline{\text { is a }}$ G-invariant retract iff $\forall g \in G, f g f=g f$.

There are often such functions in the topological case. Namely retracts onto subspaces that are invariant under al1 homeomorphisms. This relies on homeomorphisms not being globally homogeneous. The 
images might be topologically unique components of the space or of its boundary.

The semigroups generated by $G$ and $G$-invariant retracts have a very simple structure, (e.g. $\langle G \cup\{\mathrm{f}\}>=G \cup G \mathrm{fG})$.

The following describes completely automorphisms of semigroups generated by $G$ and one $G$-invariant retract.

1HEOREM 7.7. Given $\mathrm{f}$ a G-invariant retract with $\mathrm{R}=$ ran $\mathrm{f}$. Let $S=\langle G \cup\{f\}\rangle$. Let $k$ and $k^{\prime} \in G$. Necessary and sufficient conditions for $\mathrm{f} \rightarrow \mathrm{k}^{\prime} \mathrm{fk}$ to extend to an automorphism of $S$ which is the identity on $G$ are:

1) $k_{i R}^{i}=k^{-1}$

2) $\left(\mathrm{k}^{\prime} \mathrm{k}^{-1}\right)$ belongs to the normalizer of $I_{\mathrm{f}}$ in $G \times G$

Furthermore an outer automorphism is produced if and only if $\left(k^{\prime}, k^{-1}\right) \& I_{f}$.

Proof. Suppose $\hat{\phi}$ is an extension. So $\hat{\phi}(f)=\hat{\phi}(f f)$ so $k^{\prime} \mathrm{fk}=\mathrm{k}^{\prime} \mathrm{fk} \quad \mathrm{k}^{\prime} \mathrm{fk}=\mathrm{k}^{\prime} \mathrm{k} \mathrm{k}^{\prime} \mathrm{fk}$ so $\left.\mathrm{k}^{\prime} \mathrm{k}\right|_{\mathrm{R}}=\mathrm{id} \mathrm{R}_{\mathrm{R}}$ or $\mathrm{k}_{\mathrm{R}}^{\prime}=\mathrm{k}_{\mathrm{R}}^{-1}$. Since $\hat{\phi}$ is the identity on $G, I_{f}=I_{\hat{\phi} f}=I_{k^{\prime} f k}$ which by Proposition 2.3 equals $\left(\mathrm{k}^{\prime}, \mathrm{k}^{-1}\right) \circ \mathrm{I}_{\mathrm{f}} \circ\left(\mathrm{k}^{\prime}, \mathrm{k}^{-1}\right)^{-1}$, so $\left(\mathrm{k}^{\prime}, \mathrm{k}^{-1}\right)$ belongs to the normalizer of $I_{f}$. Additionally if $\hat{\phi}$ is outer then $\hat{\phi} \neq$ id so $k^{\prime} f k \neq f$, or $\left(k^{\prime}, k^{-1}\right) \notin I_{f}$. Sufficiency is shown as follows. If $\left(\mathrm{k}^{\prime}, \mathrm{k}^{-1}\right) \in \mathrm{I}_{\mathrm{f}^{\prime}}$, then $\mathrm{k}^{\prime} \mathrm{fk}=\mathrm{f}$ and the identity is the desired extension, otherwise use proposition 7.4 with $C=\left\{h f h^{-1} \mid h \in G\right\}$, and $\phi: C \rightarrow C$ given by $\phi\left(h f h^{-1}\right)=h^{\prime} \mathrm{fkh}^{-1}$. The assumption of $\left(k^{\prime}, k^{-1}\right)$ in normalizer of $I_{f^{\prime}}$ gives $\phi$ is well defined and meets the conditions of the proposition.

The following characterizes automorphisms in this case.

THEOREM 7.8. In the above case, Aut $(S) / \operatorname{Inn}(S) \cong$

$$
\left.\underline{\text { Normalizer }} \underline{\text { of }} I_{f} \text { in }\left\{\left(g^{\prime}, g\right)\left|g^{\prime}\right|_{R}=\left.g\right|_{R}\right\}\right) / I_{f^{*}}
$$

Proof. By Proposition Aut $(S) / \operatorname{Inn}(S) \cong \operatorname{Gid}(S)$ which is characterized by Theorem 7.7 .

A number of examples can be constructed using $G$-invariant retracts that have quite "rigid" graphs in the sense that the invariant $I_{f}$ is very small. A special case of Theorem 7.7 is PROPOSITION 7.9. If $\mathrm{f} \in T$ is a $G$-invariant retract with $\mathrm{R}=\underline{\text { ran }} \mathrm{f}$, and $I_{f}=\left\{\left(g_{1}, g_{2}\right)\left|g_{1}\right|_{R}=i d_{R}, g_{2}=i d\right\}$, then each $k \in G k \neq i d$ produces 
an outer automorphism of $S=\langle G \cup\{f\}\rangle$ given by $\mathrm{gfg}^{\prime} \rightarrow \mathrm{gkfk}^{-1} \mathrm{~g}^{\prime}$.

Proof. Theorem 7.7 with $\mathrm{k}^{\prime}=\mathrm{k}^{-1}$.

PROPOSITION 7.10. Let $\mathcal{C} \subset T$ and $\mathrm{R} \subset \mathrm{X} . \quad$ If $\forall f \in C$, f is a G-invariant retract with range $R$. If $\forall f \in C, I_{f}=\left\{\left(g_{1}, g_{2}\right)\left|g_{1}\right|_{R}=i d, g_{2}=i d\right\}$. Also if all inverse partitions are unique, $\left[i . e . f f^{\prime} f^{\prime} \exists x \ni f^{-1} f(x)\right.$ not homeomorphic to any $\left.f^{\prime-1} f^{\prime}\left(x^{\prime}\right)\right]$. Then $C$ and any bijection, $\phi \neq$ id satisfy Theorem 7.4 and thus produce an outer automorphism of $S=\langle C \cup G\rangle$.

EXAMPLE 7.11. Let $\mathrm{A}$ be an anulus, and $\mathrm{D}$ a disk. Take a continuous function, $f$, from $A$ onto $D$ with the property that if $g_{1}$ is a homeomorphism of $D$, and $g_{2}$ a homeomorphism of $A$, and $g_{1} \mathrm{fg}_{2}=f$ then $g_{1}$ and $\mathrm{g}_{2}$ are the identities. Let $\mathrm{x}=\mathrm{A} \cup \mathrm{D}$. Let $\mathrm{r}_{\mathrm{D}}=\mathrm{id}$ and $\mathrm{r}_{\mathrm{A}_{\mathrm{A}}}=\mathrm{f}$. Now $r$ is a G-invariant retract of $\mathrm{X}$ satisfying Proposition 7.7.

The function $\mathrm{f}$ is very rigid, it could be constructed by making its partition consist of countably many different inverse images (e.g. n-stars) dense in A with their values dense in D. Alternatively A could be mapped on to D with countably many small folds that cross in a dense manner over D. Many functions like these can be constructed that have non-equivalent inverse partitions. Proposition 7.10 would apply in this case.

The above example has $\mathrm{X}$ non-connected but this is not necessary. Consider $x$ the 3 -sphere with a solid ring and solid ball removed. This is a manifold of $\mathrm{dim}=3$ with a boundary consisting of two nonhomeomorphic components, $\mathrm{s}^{2}$ and $\mathrm{T}^{2}$. It is possible to retract $\mathrm{x}$ onto $s^{2}$. Further it can be done by a map $r$ satisfying proposition 7.9 .

\section{FURTHER DIRECTIONS}

The results showing when $S$ has the i.a.p. are of two types, when $S$ has only nice maps, or when $S$ has special maps that force the i.a.p. Even for $X=I$, these techniques produce no information when $S$ consists only of nowhere differentiable functions. I suspect that there are examples of semigroups of continuous maps on $\mathrm{X}=\mathrm{I}$ containing the group of homeomorphisms that have outer automorphisms. Whittaker's result about the group of homeomorphisms is valid for the wider class of regionally Euclidean spaces. Many of the results obtained here will have their analogues in that case. 


\section{THANKS AND ACKNOWLEDGEMENTS}

The author is most grateful to Dr. G.R. Wood for his encouragement and comments. I wish to thank Dr. R.P. Sullivan for his helpful suggestions.

\section{REFERENCES}

1. Clifford, A.H. and Preston, G.B., (1961), 'The Algebraic theory of semigroups', AMS. Mathematical Surveys 7, Chaptex 2, p.52.

2. Fine, N.J. and Schweigert, G.E., (1955), 'On the group of homeomorphisms of an arc', Ann. of Math. 62, 237 $\overline{7-253 .}$

3. Fitzpatrick, P. amd Symons, J.S.V., (1975), 'Automorphisms of transformation semigroups', Proc. Edinburgh Math. Soc. 19, $327-329$.

4. Gluskin, L.M., (1959), 'The semigroup of homeomorphic mappings of an interval', Mat. Sb. (N.S.) 49 (91), 13-18; translated in Amer. Math. Soc. Transl. 30, (1963), 273-290.

5. Gluskin, L.M., (1960), 'Automorphisms of semigroups of topological mappings', Izv. Vyss. Ucebn. Zaved. Matematika 6 (19), 62-73; translated in Amer. Math. Soc. Trans1. 36 (1964), 383-395.

6. Magil1, K.D. Jr., (1964), 'Semigroups of continuous functions', Amer. Math. Monthly 71, $984-988$.

7. Magil1, K.D. Jr., (1977), 'Homomorphisms from $S(X)$ into $S(Y)$ ', Can. J. Math. $29615-625$.

8. Magil1, K.D. Jr., and Subbiah, S., (1974), 'Green's relations for regular elements of semigroups of endomorphisms', Can. J. Math. $26,1484-1497$.

9. Sullivan, R.P., (1975), 'Automorphisms of transformation semigroups', J. Austral. Math. Soc. Ser. A 20, 77-84.

10. Whittaker, J.V., (1963), 'On isometric groups and homeomorphic spaces, Annals of Math. 78 No. 1, 74-91.

11. Wood, G.R., (1980), 'Automorphisms of semigroups of continuous functions', J. Austral. Math. Soc. Ser. A 29, 301-309.

University of Canterbury,

Christchurch,

New Zealand. 